Open Access

\title{
A tight approximate analytical framework for performance analysis of equal gain combining receiver over independent Weibull fading channels
}

\author{
Abdelmajid Bessate and Faissal El Bouanani* (D)
}

\begin{abstract}
In this paper, a method for approximating the probability distribution of sum of independent and identical Weibull random variables is adopted to analyze the performance of equal gain combiner (EGC) receiver over non-identical Weibull fading channel (WFC). Our main result is to derive a generalized expression of the probability density function (PDF) of the signal-to-noise ratio (SNR) at the EGC output in the case of non-identical WFC. Based on this PDF, accurate approximation of significant performance criteria, such as outage probability (OP), the amount of fading (AoF), and average symbol/bit error probability (ASEP/ABEP), are derived. In addition, we derived the analytical expressions for channel capacities under various adaptation policies such as optimal rate adaptation (ORA), optimal simultaneous power and rate adaptation (OPRA), channel inversion with fixed rate (CIFR), and truncated channel inversion with fixed rate (TCIFR). The proposed mathematical analysis is complemented by several numerical results and validated using Monte Carlo simulation method.

Keywords: Equal gain combining (EGC), Cumulative distribution function (CDF), Probability density function (PDF), Moment generating function (MGF), Average symbol/bit error probability (ASEP/ABEP), Adaptation policies, Fox $\mathrm{H}$-function, Meijer G-function
\end{abstract}

\section{Introduction}

Antenna diversity is one of the most practical, effective, and widely employed technique in wireless communication receivers to reduce the effects of fading and to provide increased signal strength at the receiver. Different techniques are known to combine the signals received from multiple diversity branches. The most popular diversity techniques are equal-gain combining (EGC), maximalratio combining (MRC), selection combining (SC), and a combination of MRC and SC, called generalized-selection combining (GSC). The SC receiver chooses the branch with the strongest instantaneous signal-to-noise ratio (SNR), while MRC provides optimal performance, at the expense of implementation complexity, since it requires knowledge of all channel parameters. In EGC receiver, the signals in all branches are weighted with the same

*Correspondence: f.elbouanani@um5s.net.ma

ENSIAS Mohammed V University In Rabat, Rabat, Morocco factor, irrespective of the signal amplitude. Moreover, cophasing of all input signals is needed to avoid output signal cancellation. The performance of EGC and MRC diversity receivers has been extensively conducted in many previous works for several well-known fading statistical models, such as Rayleigh, Rice, and Nakagami-assuming independent or correlative fading [1-6]. The Weibull distribution is a well-known model for describing multipath fading channels in both indoor and outdoor radio propagation environments. In [7], novel analytical expressions for the joint probability density function (PDF), moment generating function (MGF), and cumulative distribution function $(\mathrm{CDF})$ are derived for the multivariate Weibull distribution. The presented theoretical results are applied to analyze the performance of several diversity receivers such as SC, EGC, and MRC techniques operating under correlated Weibull fading channels (WFC). For these diversity receivers, several useful performance criteria such as moments of output SNR, including the amount of 
fading (AoF), and outage probability (OP) are analytically derived. Moreover, the average symbol error probability (ASEP) for several coherent and noncoherent modulation schemes is studied using moment generating function (MGF) approach. In [8], capitalizing on the general $\alpha-\mu$ fading model simple and precise closed-form approximations to the PDF and CDF of the sum of independent and identically distributed (i.i.d) Weibull variates are derived. These approximations find applicability in several wireless communications issues such as signal detection and combining, linear equalizers, intersymbol interference, and phase jitter [8]. Considering related works, C. Sagias et al. $[9,10]$, have presented a moments-based approach to analyzing the performance of dual-branch EGC and MRC receivers, operating under either independent or correlated, but not necessary identically distributed WFC. In this respect, significant performance criteria, such as average output SNR, AoF, and spectral efficiency at low power regime, are extracted in closed-forms, using the moments of the output SNR for both independent and correlative fading cases. Using the same approach of the moment, El Bouanani [11-14] has generalized this idea to $L$-branches over independent and not necessary identically distributed (i.n.i.d) Weibull fading channels for both MRC and EGC receivers. Consequently, he has derived some performance criteria such as AoF, MGF, average capacity (AC), and ASEP in closed forms. In [15], we have generalized the same idea to derive the approximate expressions of MRC performance criteria over correlated WFC.

In this paper, we propose a tight approximate expression of the output SNR PDF by generalizing the approximate CDF convolution derived in [16] to non-identical Weibull random variates (RVs). The tightness of this approximate $\mathrm{CDF}$ is proved and validated by using the KolmogorovSmirnov statistical method. Based on this approximation, closed expressions of statistical characteristic, of an EGC receiver, given in terms of well-known hypergeometric function, related to PDF, OP, and MGF are derived. Other performance criteria such as AoF, ASEP, average bit error probability (ABEP), average capacity (AC) under optimal rate adaptation with constant transmit power (ORA), capacity under optimal simultaneous power and rate adaptation (OPRA), channel inversion with fixed rate (CIFR), and truncated channel inversion with fixed rate (TCIFR) schemes are presented in tight closed-form approximation. After this introduction, Section 2 deals with the communication system model with EGC combiner over Weibull fading channels. Section 3 shows the statistical characteristics of EGC over WFC such as PDF and MGF. Section 4 presents several novel closedexpressions in terms of well-known hypergeometric functions such as that AoF, OP, AC, OPRA capacity, CIFR capacity, TCIFR capacity, and ABEP and ASEP for M-PSK and M-FSK modulation schemes. In Section 5, all results are illustrated and verified by computer simulations using Mathematica software. Finally, Section 6 contains a brief conclusion.

\section{Channel model}

We consider EGC receiver operating over slow frequencynonselective WFC. The received channel gain $R$ is expressed as

$$
R=\frac{\sum_{i=1}^{L} R_{i}}{\sqrt{L}}
$$

Where $L$ is the number of the receiver antenna and $R_{i}$ be the fading amplitude corresponding to the received signals on the $i$ th EGC-branch, assumed being i.n.i.d Weibull $\left(\beta, \omega_{i}\right) \mathrm{RV}$ with PDF:

$$
f_{R_{i}}(x)=\frac{\beta x^{\beta-1}}{\omega_{i}^{\beta}} e^{-\left(\frac{x}{\omega_{i}}\right)^{\beta}}, x \geq 0
$$

where $\beta$ and $\omega_{i}$ are the shape and scale parameters of $R_{i}$ linked with its second moment by the following expression

$$
\omega_{i}=\left[\frac{\mathbb{E}\left[R_{i}^{2}\right]}{\Gamma\left(1+\frac{2}{\beta}\right)}\right]^{\frac{1}{2}}
$$

Let $E_{s}$ and $N_{0}$ denote the average symbol energy and the thermal noise power spectral density, respectively. The instantaneous EGC output SNR is expressed in terms of instantaneous SNR $\gamma_{i}$ at the $i$ th input branch as [13]

$$
\gamma=\frac{1}{L}\left(\sum_{i=1}^{L} \sqrt{\gamma_{i}}\right)^{2}
$$

with

$$
\gamma_{i}=\frac{E_{s}}{N_{0}} R_{i}^{2}
$$

Let $\alpha_{i}=\omega_{i} \sqrt{\frac{E_{s}}{N_{0}}}$. Substituting (5) in (3), we obtain

$$
\alpha_{i}=\sqrt{\frac{\bar{\gamma}_{i}}{\Gamma\left(1+\frac{2}{\beta}\right)}}
$$

\section{Statistical characteristics}

In this section, we begin by recalling the main result of Johnson [16] regarding the derivation of a tight approximate CDF of the sum of i.i.d Weibull RVs. Furthermore, we derive the approximate PDF of output SNR at EGC receiver operating under i.i.d WFC. Our main result is to generalize this approximation to be also valid in the case of i.n.i.d WFC. 


\subsection{PDF of the sum of i.i.d Weibull RVs}

Let us bear in mind that the sum PDF of Weibull RVs is till now difficult, if not impossible, to be derived analytically. Among several works tried to approximate it, [16] is one of the most important. The author has derived a very tight approximate CDF for sum of identical $\left(\omega=\omega_{i}\right)$ Weibull RVs

$$
F_{T}(t) \approx 1-\exp \left[-\left(\frac{\sigma t}{\omega}\right)^{\beta}\right] \sum_{i=0}^{L-1} \frac{\left(\frac{\sigma t}{\omega}\right)^{\beta i}}{i !}
$$

with $T=\sum_{i=1}^{L} T_{i}, T_{i}$ is a Weibull $(\omega, \beta) \mathrm{RV}$, and $\sigma=$ $\frac{\Gamma(L+1 / \beta)}{L \Gamma(1+1 / \beta)}$.

Hence, its PDF can be approximated by deriving the expression (6)

$$
\begin{aligned}
f_{T}(t) \approx & \frac{\beta}{t} \exp \left[-\left(\frac{\sigma t}{\omega}\right)^{\beta}\right] \\
& \times\left(\sum_{i=0}^{L-1} \frac{\left[\left(\frac{\sigma t}{\omega}\right)^{\beta}\right]^{i+1}}{i !}-\sum_{i=1}^{L-1} \frac{\left(\frac{\sigma t}{\omega}\right)^{\beta i}}{(i-1) !}\right)
\end{aligned}
$$

By using the change of variable $j=i+1$ in the first sum, we obtain

$$
f_{T}(t) \simeq \frac{\sigma \beta}{\omega(L-1) !}\left(\frac{\sigma t}{\omega}\right)^{\beta L-1} \exp \left[-\left(\frac{\sigma t}{\omega}\right)^{\beta}\right]
$$

which represents the PDF of generalized Gamma (GG) RV [17] with parameters $\frac{\omega}{\sigma}, \beta L$, and $\beta$.

\subsection{PDF of output SNR at EGC receiver}

\subsubsection{Case of i.i.d WFC}

Lemma 1 Let us assume that the scale parameter of $\gamma_{i}$ is constant $\left(\alpha_{i}=\alpha=\omega \sqrt{E_{s} / N_{0}}\right)$. The square root of instantaneous $\operatorname{SNR} \gamma_{i}$ is a $\operatorname{Weibull}(\alpha, \beta)$.

Proof using the references (2) and (5), and proceeding by Jacobian transform, the PDF of the square root of $\gamma_{i}$ can be expressed as

$$
f_{\sqrt{\gamma_{i}}}(x)=\frac{\beta x^{\beta-1}}{\alpha^{\beta}} \exp \left[-\left(\frac{x}{\alpha}\right)^{\beta}\right]
$$

which concludes the Lemma proof.

Proposition 2 The output SNR at the EGC receiver over i.i.d WFC can be approximated by a $G G\left(\left[\frac{\alpha}{\sigma \sqrt{L}}\right]^{2}, \frac{\beta L}{2}, \frac{\beta}{2}\right)$ $R V$

$$
\begin{aligned}
f_{\gamma}(\gamma) \approx & \left(\frac{\sigma}{\alpha}\right)^{2} \frac{L \beta}{2(L-1) !}\left(\frac{\sigma}{\alpha} \sqrt{L \gamma}\right)^{\beta L-2} \\
& \exp \left[-\left(\frac{\sigma}{\alpha} \sqrt{L \gamma}\right)^{\beta}\right]
\end{aligned}
$$

Proof Let us denote $T=\sum_{i=1}^{L} \sqrt{\gamma_{i}}$. Adopting the expression in (8), and considering the Lemma above, the equation in (8) can be rewritten as

$$
f_{T}(t) \approx \frac{\beta}{t(L-1) !}\left(\frac{\sigma}{\alpha} t\right)^{\beta L} \exp \left[-\left(\frac{\sigma}{\alpha} t\right)^{\beta}\right]
$$

Using the expressions (4) and (11), and proceeding by Jacobian transform, we get (10) which concludes the proof of the proposition.

\subsubsection{Case of i.n.i.d Weibull RV}

It is known that two RVs are equal or very close, respectively if and only if their moments are equal or very close, respectively. Let us begin by computing both exact and approximate $n$th moment of output SNR.

\section{a. Exact nth moment of $\gamma$}

Let's define $\mathbb{E}\left[\gamma^{n}\right]=\frac{1}{L^{n}} U_{L}^{(n)}$ the exact $n$th moment of output SNR at EGC combiner. Using multinomial theorem, it can be seen that $U_{L}^{(n)}$ can be computed easily by the recurrence relation:

$$
\left\{\begin{array}{l}
\mathbb{E}\left[\gamma^{n}\right]=\frac{1}{L^{n}} U_{L}^{(n)} \\
U_{L}^{(n)}=\mathbb{E}\left[\left(\sum_{i=1}^{L} \sqrt{\gamma_{i}}\right)^{2 n}\right]=\sum_{i=0}^{2 n} C_{2 n}^{i} U_{L-1}^{(i)} \mathbb{E}\left[\gamma_{L}^{n-\frac{i}{2}}\right] \\
U_{i}^{(0)}=1, U_{1}^{(n)}=\mathbb{E}\left[\gamma_{1}^{n}\right]
\end{array}\right.
$$

where the $n$th moment of $\gamma_{i}$ is given by [14] as

$$
\mathbb{E}\left[\gamma_{i}^{n}\right]=\Gamma\left(1+\frac{2 n}{\beta}\right)\left(\frac{\bar{\gamma}_{i}}{\Gamma\left(1+\frac{2}{\beta}\right)}\right)^{n}
$$

b. Approximate nth moment of $G G R V$

Let $\mu$ be a real positive and $Z$ be a $\operatorname{GG}\left(\mu, \frac{\beta L}{2}, \frac{\beta}{2}\right)$ $\mathrm{RV}$. The $n$th moment of $Z$ can be computed as

$$
\mathbb{E}\left[Z^{n}\right]=\int_{0}^{+\infty} x^{n} f_{Z}(x) d x
$$

Substituting the PDF (10) into (14), and taking the change of variable $y=\left(\frac{x}{\mu}\right)^{\frac{\beta}{2}}$, the $n$th moment of $Z$ can be expressed briefly as

$$
\mathbb{E}\left[Z^{n}\right]=\mu^{n} \frac{\Gamma\left(L+\frac{2 n}{\beta}\right)}{(L-1) !}
$$

Proposition 3 The output SNR at the EGC receiver over i.n.i.d WFC can be also approximated by a $G G\left(\theta^{2}, \frac{\beta L}{2}, \frac{\beta}{2}\right)$ $R V$

$$
f_{\gamma}(\gamma) \approx \lambda \exp \left[-\left(\frac{\gamma}{\theta^{2}}\right)^{\frac{\beta}{2}}\right]\left(\frac{\gamma}{\theta^{2}}\right)^{\frac{\beta}{2}\left(L-\frac{2}{\beta}\right)}
$$


where

$$
\theta=\frac{\rho}{\sigma \sqrt{L}}
$$

with

$$
\rho=\frac{\sum_{i=1}^{L} \alpha_{i}}{L}=\frac{\sum_{i=1} \sqrt{\bar{\gamma}_{i}}}{L \Gamma^{\frac{1}{2}}\left(1+\frac{2}{\beta}\right)}
$$

and

$$
\lambda=\frac{\beta}{2 \theta^{2}(L-1) !}
$$

Proof To compare the exact and approximate $n$th moment, we will proceed by two approaches. When $\beta$ is sufficiently large, an analytical approach, based on approximation at infinity of both exact and approximate $n$th moments, is used to prove equality between them. Otherwise, plotting the two moment curves, for several values of $\varphi$ and $\beta$, will be useful to show the closeness between the two expressions.

\section{a. Analytical approach}

Let $\gamma_{A}$ denote the GG RV that approximates output SNR $\gamma$ at EGC receiver. Substituting (18) in (17) and substituting (17) in (15) by taking $\mu=\theta^{2}$, the $n$th exact moment of $\gamma_{A}$ can be rewritten as

$$
\begin{aligned}
\mathbb{E}\left[\gamma_{A}^{n}\right]= & \frac{(L !)^{2 n} \Gamma^{2 n}\left(1+\frac{1}{\beta}\right) \Gamma\left(L+\frac{2 n}{\beta}\right)}{(L-1) ! L^{3 n} \Gamma^{2 n}\left(L+\frac{1}{\beta}\right) \Gamma^{n}\left(1+\frac{2}{\beta}\right)} \\
& \times\left(\sum_{i=1}^{L} \sqrt{\bar{\gamma}_{i}}\right)^{2 n}
\end{aligned}
$$

Now, using the identity ([21],/06.05.06.0021.01) for great values of $\beta$, we obtain the following approximation:

$$
\begin{aligned}
\Gamma\left(L+\frac{2 n}{\beta}\right) & \sim(L-1) !\left(1+\Psi(L) \frac{2 n}{\beta}\right) \\
\Gamma^{2 n}\left(L+\frac{1}{\beta}\right) & \sim\left((L-1) !\left(1+\Psi(L) \frac{1}{\beta}\right)\right)^{2 n} \\
& \sim[(L-1) !]^{2 n}\left(1+\Psi(L) \frac{2 n}{\beta}\right) \\
\Gamma^{2 n}\left(1+\frac{1}{\beta}\right) & \sim\left(1+\Psi(1) \frac{2 n}{\beta}\right) \\
\Gamma^{n}\left(1+\frac{2}{\beta}\right) & \sim\left(1+\Psi(1) \frac{2 n}{\beta}\right)
\end{aligned}
$$

where $\Psi($.$) is the digamma function that represents$ the first derivation of Gamma function.

Substituting (21), (22), (23), and (24) into (20), we deduce that

$$
\mathbb{E}\left[\gamma_{A}^{n}\right] \approx \frac{1}{L^{n}}\left(\sum_{i=1}^{L} \sqrt{\bar{\gamma}_{i}}\right)^{2 n},
$$

On the other hand, the $n$th exact moment of the output SNR can be expressed by using (12) as

$$
\begin{aligned}
\mathbb{E}\left[\gamma^{n}\right]= & \frac{1}{L^{n}} \sum_{k_{1}+. .+k_{L}=2 n} \frac{2 n !}{k_{1} ! \ldots k_{L} !} \prod_{i=1}^{L} \Gamma\left(1+\frac{k_{i}}{\beta}\right) \\
& \times\left(\frac{\bar{\gamma}_{i}}{\Gamma\left(1+\frac{2}{\beta}\right)}\right)^{\frac{k_{i}}{2}}
\end{aligned}
$$

Based on the same approximation used previously, we can note that

$$
\begin{aligned}
& \Gamma\left(1+\frac{k_{i}}{\beta}\right) \sim\left(1+\Psi(1) \frac{k_{i}}{\beta}\right) \\
& \Gamma^{\frac{k_{i}}{2}}\left(1+\frac{2}{\beta}\right) \sim\left(1+\Psi(1) \frac{k_{i}}{\beta}\right)
\end{aligned}
$$

By replacing (28) and (27) into (26), it follows that the $n$th exact moment can be approximated, for great values of $\beta$, by

$$
\mathbb{E}\left[\gamma^{n}\right] \approx \frac{1}{L^{n}}\left(\sum_{i=1}^{L} \sqrt{\bar{\gamma}_{i}}\right)^{2 n}
$$

Consequently, for each natural integer $n$, the exact and approximate $n$th moments can be approximated by the same expression as given by (25) and (29). Thereby, the two RV $\gamma$ and $\gamma_{A}$ are very close.

\section{b. Curve-based approach}

Taking into account that it is very difficult to upper bound the error between exact and approximate moment, we will opt for a graphical approach by plotting the curves of the moments versus the power delay profile $\varphi$ and the shape parameter $\beta$. See the evaluation section.

From the results depicted in these figures, we deduct that the fifth first exact and approximate moments are very close, which concludes the proof of the proposition.

$$
\mathbb{E}\left[\gamma^{n}\right]_{A} \simeq \mathbb{E}\left[\gamma^{n}\right], 1 \leq n \leq 5
$$




\subsection{MGF of the output SNR $\gamma$}

Proposition 4 The moment generating function of $\gamma$ for EGC receiver over i.n.i.d Weibull fading channels can be tightly approximated for any positive real $t$ by

$$
M_{\gamma}(t) \simeq \frac{\lambda}{t} H_{1,1}^{1,1}\left(\begin{array}{l|c}
\left(\frac{1}{\theta^{2} t}\right)^{\beta / 2} & \left(0, \frac{\beta}{2}\right) \\
\left(L-\frac{2}{\beta}, 1\right)
\end{array}\right)
$$

Proof The moment generating function (MGF) of $\gamma$ is by definition $M_{\gamma}(t)=\int_{0}^{\infty} \exp [-t \gamma] f_{\gamma}(\gamma) d \gamma$, replacing the PDF in reference (16) by its term, we obtain

$$
\begin{aligned}
M_{\gamma}(t)= & \lambda \int_{0}^{\infty} \exp [-t \gamma] \exp \left[-\left(\frac{\gamma}{\theta^{2}}\right)^{\frac{\beta}{2}}\right] \\
& \times\left(\frac{\gamma}{\theta^{2}}\right)^{\frac{\beta}{2}\left(L-\frac{2}{\beta}\right)} d \gamma
\end{aligned}
$$

Now, rewriting the PDF in (16) and the exponential function as a Meijer G-function ([21], /07.34.03.0228.01) we obtain the following expression

$$
M_{\gamma}(t)=\lambda \int_{0}^{\infty} G_{0,1}^{1,0}\left(t \gamma \mid \begin{array}{l}
- \\
0
\end{array}\right) G_{0,1}^{1,0}\left(\left(\frac{\gamma}{\theta^{2}}\right)^{\frac{\beta}{2}} \mid \begin{array}{c}
- \\
L-2 / \beta
\end{array}\right) d \gamma
$$

Using the equality ([21], /07.34.21.0012.01) the MGF can be written as mentioned in (30).

\section{Performance criteria}

\subsection{Outage probability of the output SNR $\gamma$}

Let $\gamma_{\text {th }}$ be the minimum SNR threshold that guarantees the reliable communication and having the corresponding channel not in outage. Regarding the CDF and PDF of GG RV given by (6) and (8), respectively, and considering the expression PDF (16), the outage probability can be expressed as

$$
P_{\text {out }}=F_{\gamma}\left(\gamma_{\text {th }}\right)=1-\exp \left[-\left(\frac{\gamma_{\text {th }}}{\theta^{2}}\right)^{\frac{\beta}{2}}\right] \sum_{i=0}^{L-1} \frac{\left(\frac{\gamma_{\text {th }}}{\theta^{2}}\right)^{\frac{\beta i}{2}}}{i !}
$$

with $\theta$ is expressed in (17).

\subsection{Amount of fading (AoF)}

The amount of fading (AoF) is a simple measure for the performance of a diversity system which is defined as

$$
\eta_{\gamma}=\frac{\mu_{2}}{\mu_{1}^{2}}-1
$$

with $\mu_{2}$ and $\mu_{1}$ are respectively the first and second moment of the output SNR $\gamma$.
Let's compute the first and second approximate moment of $\gamma$ from (15) and substituting it into (32). The AoF for an EGC combiner operating under i.n.i.d WFC can be approximated by

$$
\eta_{\gamma} \simeq \frac{\Gamma\left(L+\frac{4}{\beta}\right)(L-1) !}{\Gamma^{2}\left(L+\frac{2}{\beta}\right)}-1
$$

\subsection{Capacity under ORA policy}

The channel capacity under ORA policy $\langle C\rangle_{O R A}$ can be obtained, in the case of flat-fading channel, by averaging over the distribution of the received SNR $\gamma$ that of AWGN channel

$$
\begin{aligned}
\langle C\rangle_{\text {ORA }} & =\mathbb{E}\left[\log _{2}(1+\gamma)\right] \\
& =\int_{0}^{+\infty} \log _{2}(1+\gamma) f_{\gamma}(\gamma) d \gamma
\end{aligned}
$$

The capacity under the ORA scheme is also called ergodic capacity. Rewriting the expression of the approximate PDF given by (16) by Meijer G-function and substituting it with ([21], /07.34.03.0456.01) into (34), and using the equality ([21], /07.34.21.0012.01), the $\langle C\rangle_{\text {ORA }}$ under EGC diversity over i.n.i.d Weibull fading channels is tightly approximated by a Fox $\mathrm{H}$-function

$$
\langle C\rangle_{\mathrm{ORA}} \simeq \frac{\lambda}{\ln 2} H_{2,3}^{3,1}\left(\theta^{-\beta} \mid \begin{array}{l}
\left(-1, \frac{\beta}{2}\right),\left(0, \frac{\beta}{2}\right) \\
\left(L-\frac{2}{\beta}, 1\right),\left(-1, \frac{\beta}{2}\right),\left(-1, \frac{\beta}{2}\right)
\end{array}\right)
$$

\subsection{Capacity under OPRA policy}

The capacity under OPRA policy can improve the ergodic capacity, By varying the rate and power. Let $\gamma_{0}$ denotes the optimal threshold SNR below which no data is transmitted. Under this scheme, the capacity is known as [18]

$$
\langle C\rangle_{\text {OPRA }}=\int_{\gamma_{0}}^{+\infty} \log _{2}\left(\frac{\gamma}{\gamma_{0}}\right) f_{\gamma}(\gamma) d \gamma
$$

Proposition 5 The capacity under OPRA policy of EGC receiver operating under i.n.i.d WFC is approximated by

$$
\langle C\rangle_{O P R A} \simeq \frac{2}{\beta \ln 2} \sum_{i=0}^{L-1} \frac{\Gamma\left(i, x_{0}\right)}{i !}
$$

where $x_{0}=\left(\frac{\gamma_{0}}{\theta^{2}}\right)^{\frac{\beta}{2}}$ and $\Gamma$ (., .) denote the upper incomplete gamma function. 
Proof Performing some algebraic operations and using the integration by parts, the integral in (36) can be rewritten as

$$
\begin{aligned}
\langle C\rangle_{\text {OPRA }}= & \frac{1}{\ln (2)}\left[\ln (\gamma)\left(F_{\gamma}(\gamma)-1\right)\right]_{\gamma_{0}}^{\infty} \\
& -\frac{1}{\ln (2)} \int_{\gamma_{0}}^{\infty} \frac{1}{\gamma}\left(F_{\gamma}(\gamma)-1\right) d \gamma \\
& -\log _{2}\left(\gamma_{0}\right) \int_{\gamma_{0}}^{\infty} f_{\gamma}(\gamma) d \gamma
\end{aligned}
$$

Substituting the expression (31) into the first term of the above equation, and seeing that $\lim _{\gamma \rightarrow \infty} \frac{\ln (\gamma)}{\gamma}\left(\frac{\gamma}{\theta^{2}}\right)^{\frac{\beta}{2} i+1}$ $e^{-\left(\frac{\gamma}{\theta^{2}}\right)^{\frac{\beta}{2}}}=0$ for all natural integer, $i \leq L-1$, the first expression in (38) can be reduced to

$$
\begin{aligned}
& \frac{1}{\ln (2)}\left[\ln (\gamma)\left(F_{\gamma}(\gamma)-1\right)\right]_{\gamma_{0}}^{\infty} \\
& \quad=-\log _{2}\left(\gamma_{0}\right)\left(F_{\gamma_{0}}\left(\gamma_{0}\right)-1\right)
\end{aligned}
$$

Now, replacing (31) into the second term of Eq. (38), and using the change of variable $t=\left(\frac{\gamma}{\theta^{2}}\right)^{\frac{\beta}{2}}$, this term can be expressed as

$$
\begin{aligned}
\frac{-1}{\ln (2)} & \int_{\gamma_{0}}^{\infty} \frac{1}{\gamma}\left(F_{\gamma}(\gamma)-1\right) d \gamma \\
& =\sum_{i=0}^{L-1} \frac{2}{\beta \ln (2) i !} \int_{u_{0}}^{\infty} t^{i-1} \exp (-t) d t \\
& =\frac{2}{\beta \ln (2)} \sum_{i=0}^{L-1} \frac{\Gamma\left(i, u_{0}\right)}{i !}
\end{aligned}
$$

On the other hand, the last term of (38) can be easily expressed as

$$
-\log _{2}\left(\gamma_{0}\right) \int_{\gamma_{0}}^{\infty} f_{\gamma}(\gamma) d \gamma=\log _{2}\left(\gamma_{0}\right)\left(F_{\gamma_{0}}\left(\gamma_{0}\right)-1\right)
$$

Now, substituting (39), (40), and (41) into (38), we obtain the expression (37) which concludes the proposition's proof.

\subsubsection{Optimal cutoff SNR $\gamma_{0}$}

The optimal cutoff SNR $\gamma_{0}$ level, below which data transmission is suspended, must satisfy [23]

$$
\int_{\gamma_{0}}^{+\infty}\left(\frac{1}{\gamma_{0}}-\frac{1}{\gamma}\right) f_{\gamma}(\gamma) d \gamma=1
$$

Since no data is sent when $\gamma<\gamma_{0}$, the optimal policy suffers a probability of outage $P_{\text {out }}$, equal to the probability of no transmission, given by

$$
P_{\text {out }}=1-\int_{\gamma_{0}}^{+\infty} f_{\gamma}(\gamma) d \gamma
$$

Substituting (16) in (42), and using the same change of variable given above, we find that $\gamma_{0}$ must satisfy

$$
\begin{aligned}
& \frac{2 \lambda}{\beta \theta^{2} \gamma_{0}} \int_{x_{0}}^{+\infty} x^{L-1} \exp [-x] d x-\frac{2 \lambda}{\beta} \int_{x_{0}}^{+\infty} x^{L-\frac{2}{\beta}-1} \\
& \quad \exp [-x] d x=1
\end{aligned}
$$

Now, replacing (19) into (43), $x_{0}$ must satisfy

$$
f\left(x_{0}\right)=0
$$

where the function $f$ is defined by

$$
f(y)=\frac{\Gamma(L, y) y^{-2 / \beta}}{\theta^{2}}-\frac{\Gamma\left(L-\frac{2}{\beta}, y\right)}{\theta^{2}}-\Gamma(L)
$$

Using the derivative of the upper incomplete gamma function, we obtain $\frac{\partial f(y)}{\partial y}=-\frac{2}{\beta \theta^{2}} \Gamma(L, y) y^{\frac{-2}{\beta}-1}<0$ for all $y \geq 0$. Moreover, $\lim _{y \rightarrow 0^{+}} f(y)=+\infty$ and $\lim _{y \rightarrow+\infty} f(y)=$ $-\Gamma(L)<0$. Thus, we conclude that there is a unique $x_{0}$, consequently a unique $\gamma_{0}$, for which $f\left(x_{0}\right)=0$. Besides, the value of $\gamma_{0}$ can be calculated, using any calculation software, by solving (44).

\subsection{Capacity under CIFR policy}

Proposition 6 The capacity under CIFR policy for EGC combiner operating under i.n.i.d WFC is approximated by

$$
\langle C\rangle_{C I F R} \simeq \log _{2}\left[1+\frac{\theta^{2}(L-1) !}{\Gamma\left(L-\frac{2}{\beta}\right)}\right]
$$

Proof The channel capacity under CIFR policy is given by

$$
\begin{aligned}
\langle C\rangle_{\mathrm{CIFR}} & =\log _{2}\left[1+\frac{1}{\mathbb{E}\left[\gamma^{-1}\right]}\right] \\
& =\log _{2}\left[1+\frac{1}{\int_{0}^{+\infty} \gamma^{-1} f_{\gamma}(\gamma) d \gamma}\right]
\end{aligned}
$$

Using (16) and the same change of variable used above, we obtain

$$
\begin{aligned}
\int_{0}^{+\infty} \gamma^{-1} f_{\gamma}(\gamma) d \gamma= & \frac{1}{\theta^{2}(L-1) !} \int_{0}^{+\infty} u^{L-\frac{2}{\beta}-1} \\
& \exp (-u) d u \Gamma=\left(L-\frac{2}{\beta}\right)
\end{aligned}
$$

Thus, by substituting (48) into (47), the expression in (46) can be easily derived. 
Table 1 Values of $\varrho$ and $\delta$ for some signaling constellations

\begin{tabular}{llll}
\hline Modulation & $M$ & $\varrho$ & $\delta$ \\
\hline BPSK & 2 & $1 / 2$ & 1 \\
BFSK & 2 & $1 / 2$ & $1 / 2$ \\
$M$-PSK & $\geq 4$ & 1 & $\sin ^{2}(\pi / M)$ \\
$M$-FSK & $\geq 4$ & $(M-1) / 2$ & $1 / 2$ \\
\hline
\end{tabular}

\subsection{Capacity under TCIFR policy}

Proposition 7 The capacity under TCIFR policy of EGC receiver over i.n.i.d WFC is approximated by

$$
\begin{aligned}
\langle C\rangle_{\text {TCIFR }} \simeq & \log _{2}\left[1+\frac{\theta^{2}(L-1) !}{\Gamma\left(L-\frac{2}{\beta}\right)}\right] \\
& \times e^{-\left(\frac{\gamma^{*}}{\theta^{2}}\right)^{\frac{\beta}{2}}} \sum_{i=0}^{L-1} \frac{\left(\frac{\gamma^{*}}{\theta^{2}}\right)^{\frac{\beta}{2} i}}{i !}
\end{aligned}
$$

where $\gamma^{*}$ is a fixed cutoff fade depth.

Proof The capacity with truncated channel inversion and fixed rate policy is given by [18] as

$$
\begin{aligned}
\langle C\rangle_{\mathrm{TCIFR}}= & \log _{2}\left[1+\frac{1}{\int_{0}^{+\infty} \gamma^{-1} f_{\gamma}(\gamma) d \gamma}\right] \\
& \times\left(1-P_{\text {out }}\left(\gamma^{*}\right)\right)
\end{aligned}
$$

From (50), it is obvious that $\langle C\rangle_{\mathrm{TCIFR}}=$ $\langle C\rangle_{\mathrm{CIFR}}\left(1-P_{\text {out }}\left(\gamma^{*}\right)\right)$. Then by substituting (31) and (46) into (50), we easily obtain (49).

\subsection{Average symbol error probability (ASEP)}

Proposition 8 The ASEP of various M-ary modulation with EGC receiver over flat i.n.i.d Weibull multipath fading channels is closely approximated by

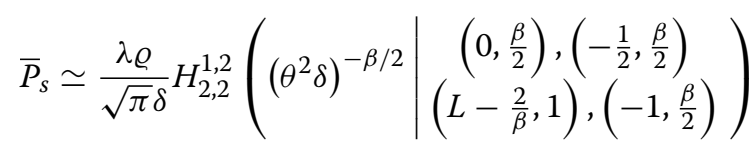

where $\varrho$ and $\delta$ are summarized in Table 1.

Proof The ASEP for $M$-ary modulation schemes over fading channel is expressed by its definition as

$$
\bar{P}_{s}=\int_{0}^{+\infty} P_{\text {se }}(\gamma) f_{\gamma}(\gamma) d \gamma
$$

where the instantaneous $\operatorname{ASEP} P_{\text {se }}(\gamma)$ is given in terms of the complementary error function as

$$
P_{\text {se }}(\gamma)=\varrho . e r f c(\sqrt{\delta \gamma})
$$

then, by rewriting the PDF in (16) and the equation in (53) by Meijer G-function the ASEP in (52) can be tightly approximated by

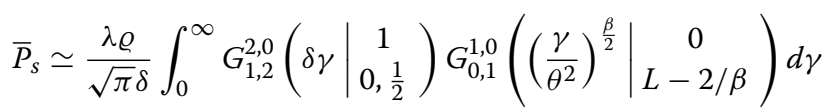

Now, using the identity ([21], /07.34.21.0012.01) we obtain (51) which concludes the proof of the proposition.

\subsection{Average bit error probability (ABEP)}

Proposition 9 The ABEP for various modulation schemes, which are cited in Table 1, can be approximated by

$$
\bar{P}_{e} \simeq \frac{\lambda \varrho}{2 \sqrt{\pi} \delta} H_{2,2}^{1,2}\left(\begin{array}{l|c}
\left(\theta^{2} \delta\right)^{-\beta / 2} & \begin{array}{c}
\left(0, \frac{\beta}{2}\right),\left(-\frac{1}{2}, \frac{\beta}{2}\right) \\
\left(L-\frac{2}{\beta}, 1\right),\left(-1, \frac{\beta}{2}\right)
\end{array}
\end{array}\right)
$$

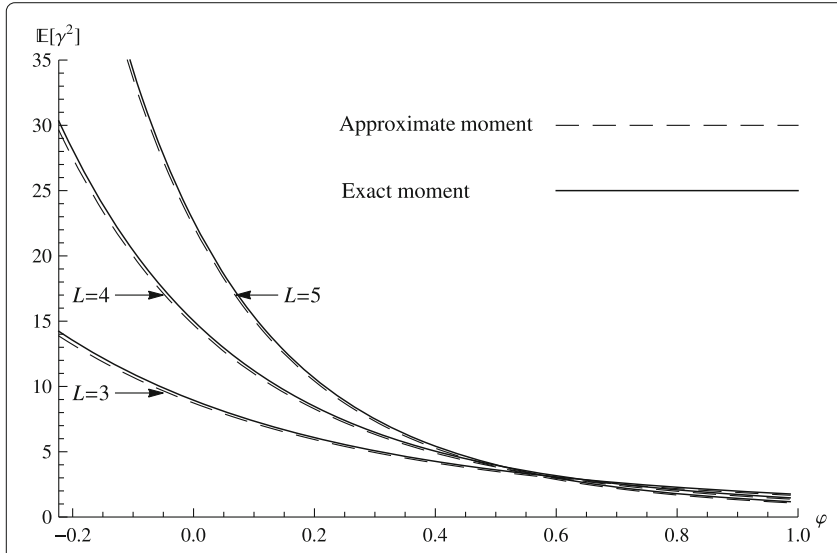

(a) Second moment

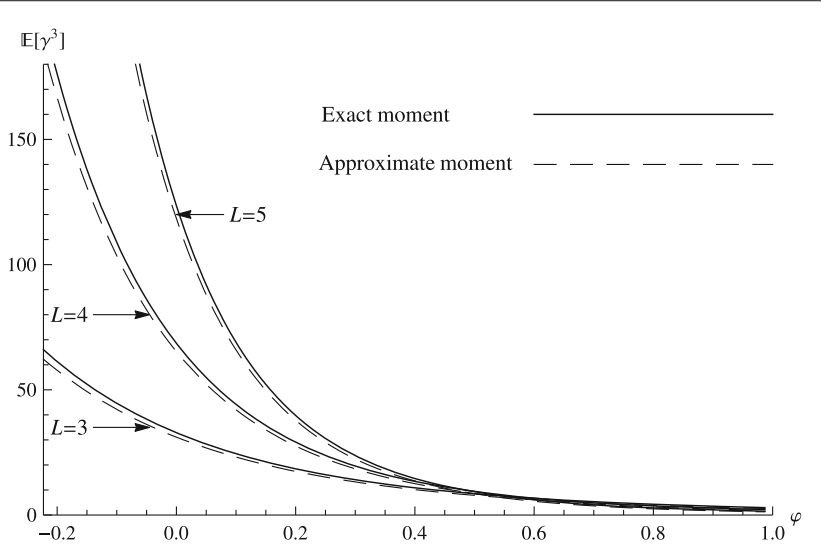

(b) Third moment

Fig. 1 Approximate and exact second and third moment of the output SNR versus $\varphi$ 


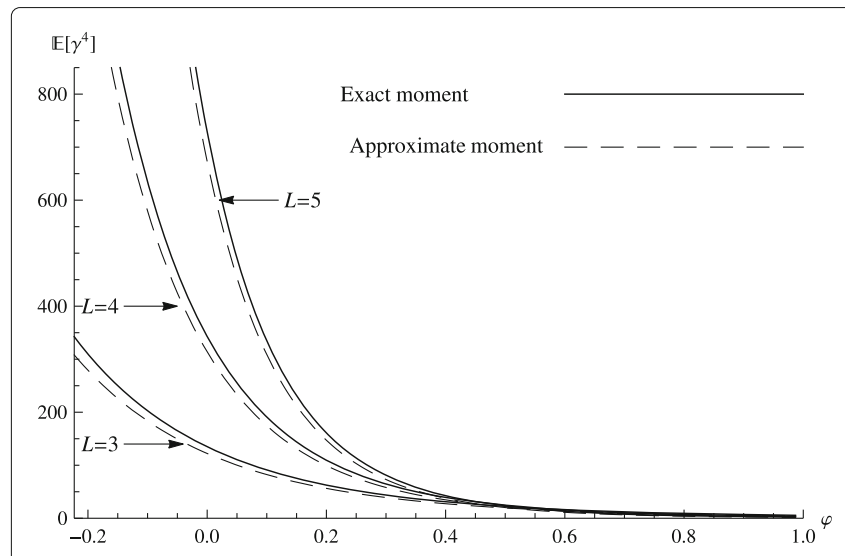

(a) Fourth moment

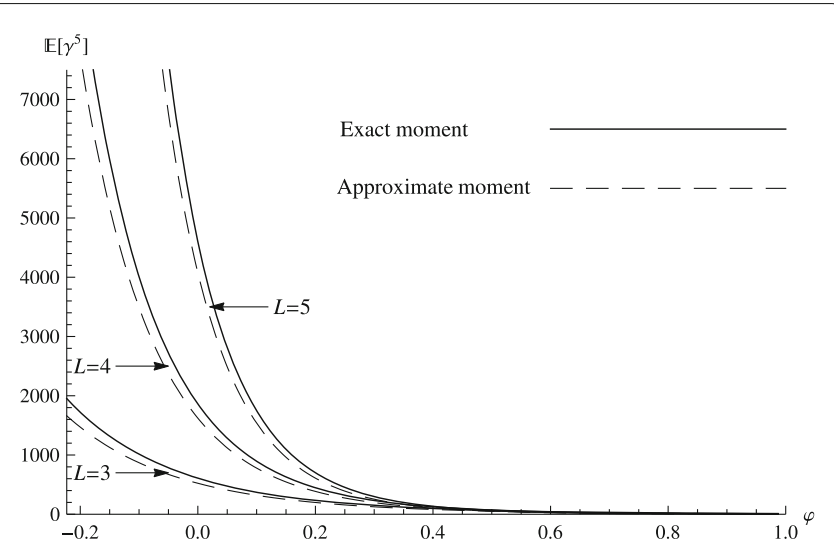

(b) Fifth moment

Fig. 2 Approximate and exact fourth and fifth moment of the output SNR versus $\varphi$

Proof By definition, the ABEP can be expressed in terms of MGF as ([19], Eq. (19))

$$
\bar{P}_{e}=\frac{\varrho}{\pi} \int_{0}^{\pi / 2} M \gamma\left(\frac{\delta}{\sin ^{2}(\phi)}\right) d \phi
$$

By considering the expression of MGF given in (30), and rewriting it in terms of Mellin-Barnes integral, we get

$$
\begin{gathered}
\bar{P}_{e} \simeq \frac{\lambda \varrho}{2 \pi^{2} j \delta} \oint_{C} \Gamma\left(1-\frac{\beta}{2} s\right) \Gamma\left(L-\frac{2}{\beta}+s\right) \\
\times\left(\frac{1}{\theta^{2} \delta}\right)^{-\frac{\beta s}{2}} \int_{0}^{\pi / 2} \sin ^{2-\beta s}(\phi) d \phi d s
\end{gathered}
$$

where $C$ is a complex contour of integration and $\int_{0}^{\pi / 2} \sin ^{2-\beta s}(\phi) d \phi$ can be expressed, when $\operatorname{Re}(\beta s)<3$, in terms of Beta function as ([21], /06.18.07.0002.01)

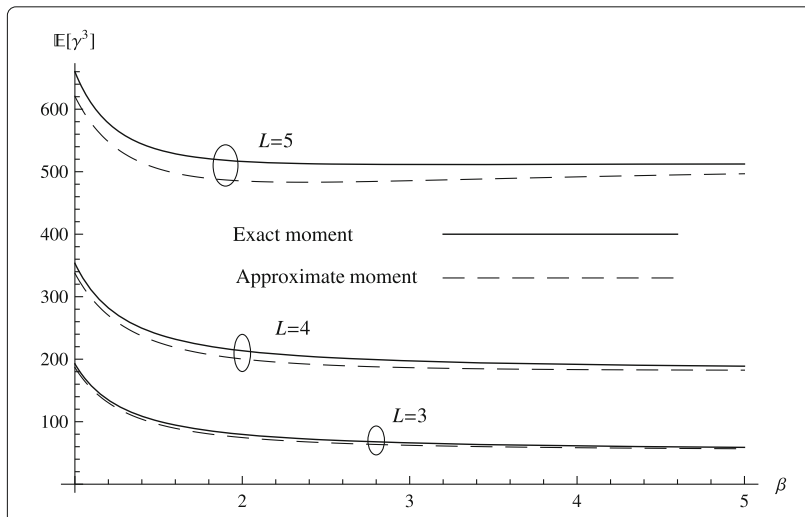

Fig. 3 Approximate and exact third moment of the output SNR versus $\beta$

$$
\begin{aligned}
\int_{0}^{\pi / 2} \sin ^{2-\beta s}(\phi) d \phi & =\frac{1}{2} B\left(\frac{3-\beta s}{2}, \frac{1}{2}\right) \\
& =\frac{\sqrt{\pi}}{2} \frac{\Gamma\left(\frac{3}{2}-\frac{\beta}{2} s\right)}{\Gamma\left(2-\frac{\beta}{2} s\right)}
\end{aligned}
$$

Now, by substituting (58) into (57), we obtain the expression (55)

\section{Evaluation}

In this section, all the analytical expressions are evaluated using Mathematica software. In addition, our approximate PDF and most of other results are validated using numerical Monte Carlo simulations from (4) by generating $L \times$ $10^{7}$ Weibull-distributed random values. Without loss of generality, we have supposed an exponentially decaying power delay profile (PDP) $\bar{\gamma}_{i} / \bar{\gamma}_{1}=\exp [-\varphi(i-1)]$, where $\varphi$ is the average fading power decay factor [22], and $\bar{\gamma}_{1}=1$ for all figures except Figs. 10 and 15. The value of $\beta$ is assumed to be the same for each receiver branch.

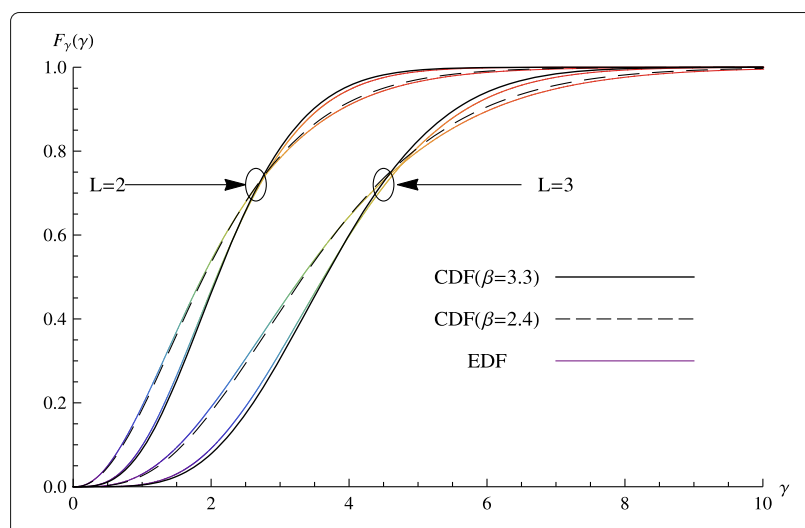

Fig. 4 Approximate and simulated CDF of the SNR at the EGC receiver 


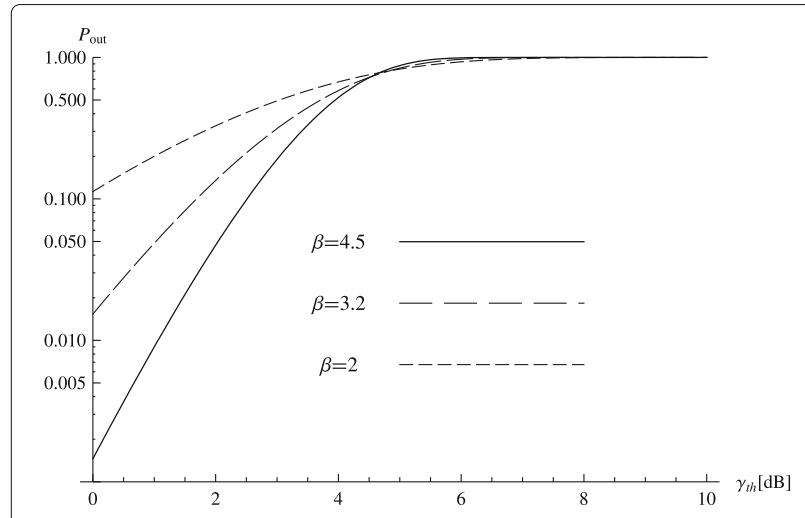

Fig. 5 Outage probability of L-branch EGC diversity

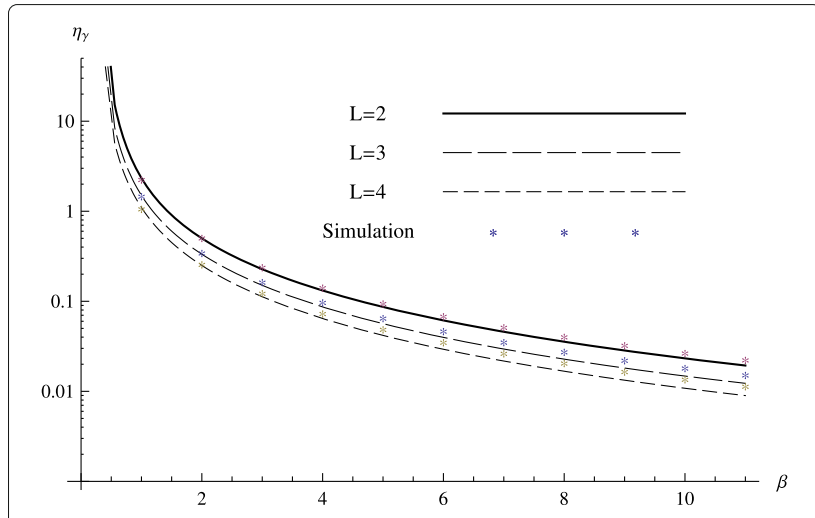

Fig. 8 Analytical and simulated AoF for L-branch EGC receiver
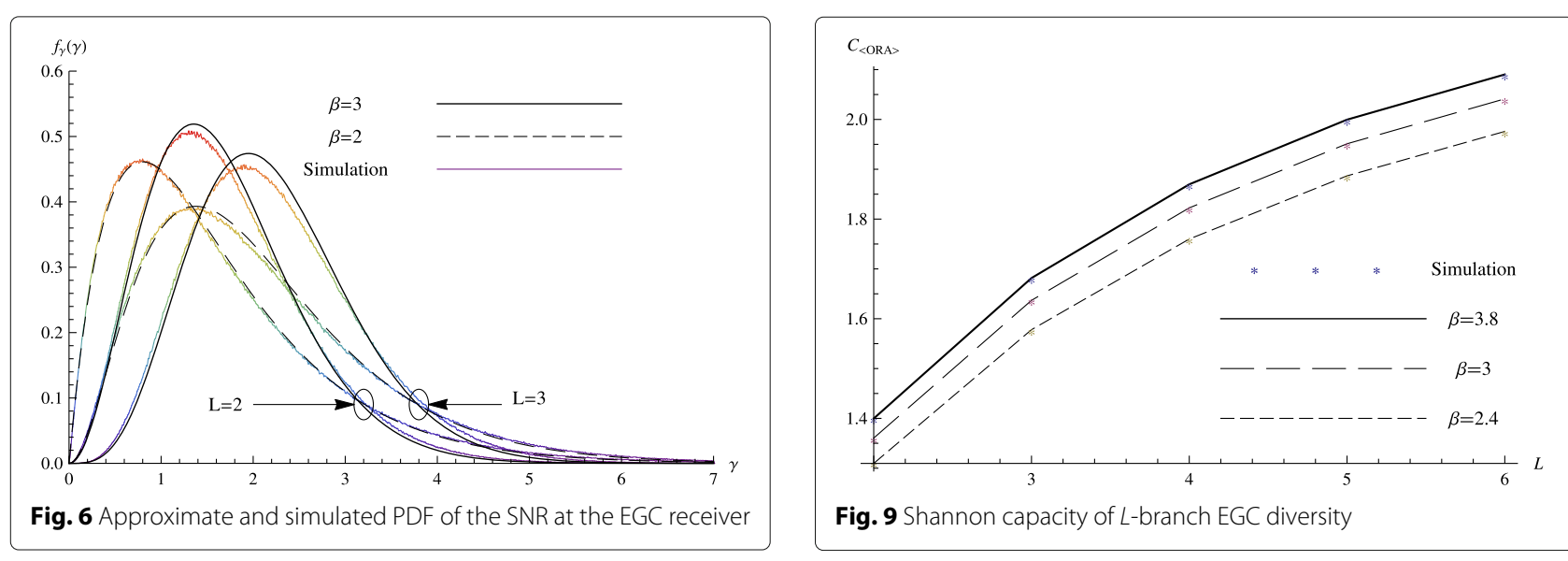

Fig. 9 Shannon capacity of L-branch EGC diversity

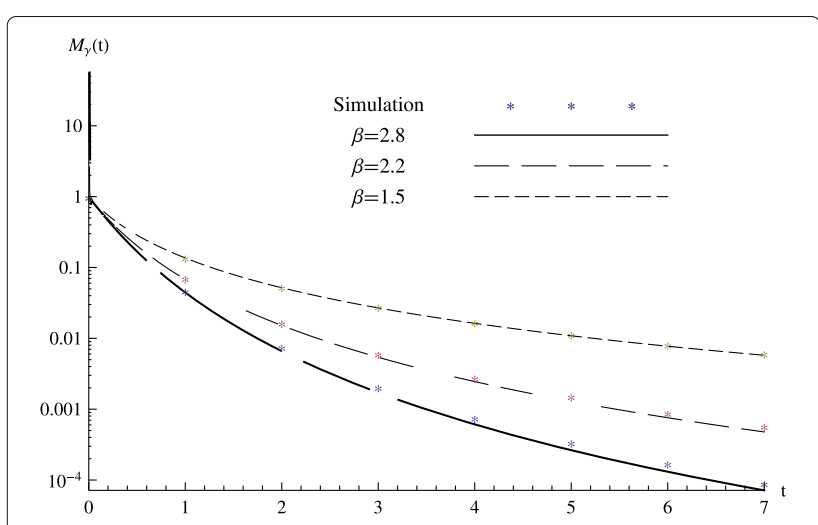

Fig. 7 MGF of the output received SNR at the EGC receiver

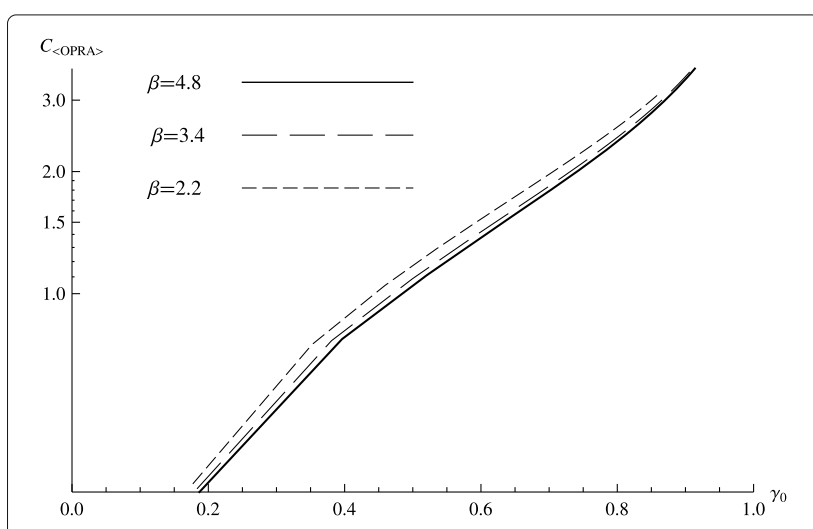

Fig. 10 OPRA capacity of 3-branch EGC diversity versus $\gamma_{0}$ 


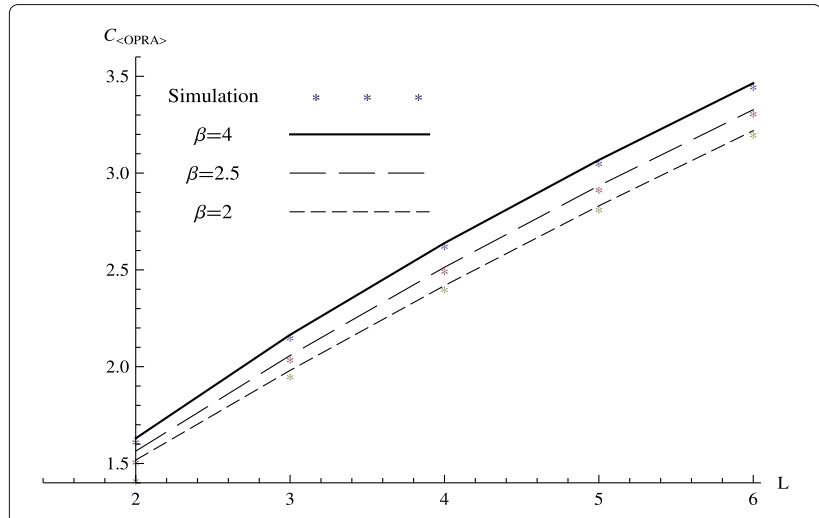

Fig. 11 OPRA capacity of 3-branch EGC combiner versus $L$

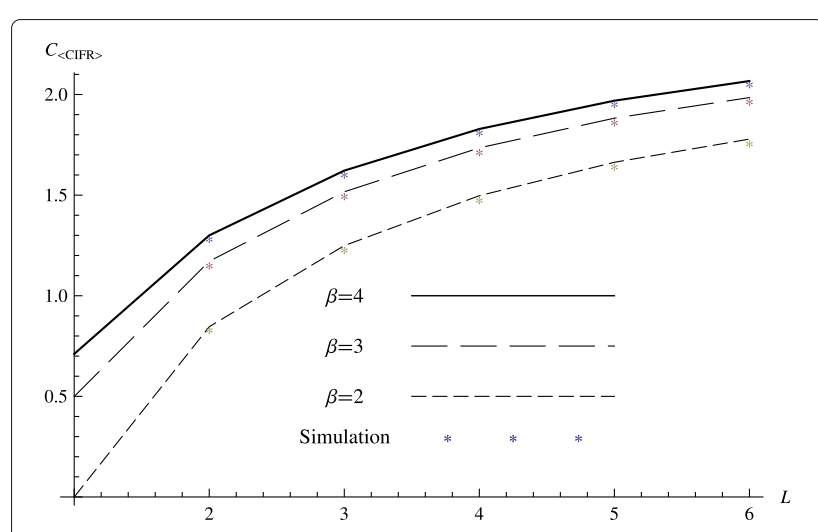

Fig. 13 CIFR capacity of L-branch EGC receiver
The two first figures depict the exact and approximate $n$th moments of output SNR versus $\varphi$ for $\beta=3$ and various values of $n$. We notice from these curves that the two moments are almost confused (Figs. 1 and 2). In addition, for $\varphi$ tending towards 1 , all curves will be confused regardless of $\beta$ and $L$.

Figure 3 shows the exact and approximate third moment of output SNR versus $\beta$ for $\varphi=-0.223$. The main remark is that the two moments are nearly confused for $L=3$ and begin to separate when $L$ becomes large.

Figure 4 shows the two curves, that of approximate CDF, and that of the empirical distribution function (EDF). The Kolmogorov-Smirnov statistic quantifies the distance between the EDF and the approximated CDF. From this figure, we deduce that the distance between the two curves is almost null, which proves the tightness of our approximation.

Figure 5 shows the progression of the outage probability $P_{\text {out }}$, computed analytically from (31), versus $\gamma_{\text {th }}$ for four branches EGC receiver, and various values of $\beta$. It can be seen that the decrease in outage probability achieved by increasing the shape parameter $\beta$ and then the system

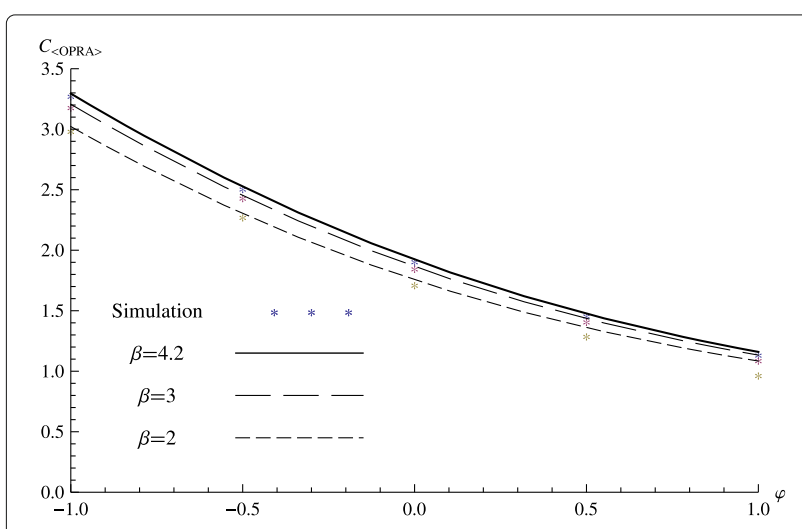

Fig. 12 OPRA capacity of 3-branch EGC combiner versus $\varphi$ becomes more reliable. Besides, as $\gamma_{\text {th }}$ increases, $P_{\text {out }}$ reaches 1 .

Figure 6 presents both the approximate and the simulated PDF of output SNR versus $\gamma$ for double-branch EGC receiver and triple one. The theoretical curves are traced from (17). Furthermore, for simulated curves, the SNR range [0,7] is divided into 100 subintervals of equal length. What is evident from both curves is that our new analytical expression of PDF is very close to its simulation.

Figure 7 displays both the approximate and the simulated MGF of the output SNR. The approximate MGF given by (30) is plotted versus $t$ for many values of $\beta$ ( $\beta=$ $2.8, \beta=2$, and $\beta=1.5$ ), and $L=3$. The curves show that the greater is $\beta$, the smaller is the MGF, and its value at $t=0$ is equal to 1 for any parameter values. What is evident from both curves is that the analytical expression is nearly similar to the simulation of the SNR MGF.

Figure 8 presents both simulated and approximate AoF given by (33). The curves of AoF are plotted versus $\beta$ for several values of $L$. It can be seen that $\eta_{\gamma}$ decreases as $\beta$ and $L$ increases, which explains the usefulness of several branches at the receiver.

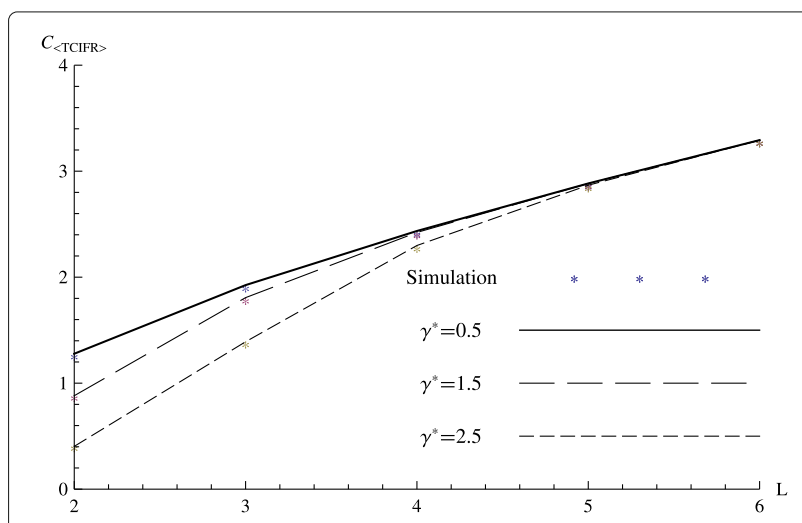

Fig. 14 TCIFR capacity of L-branch EGC combiner 


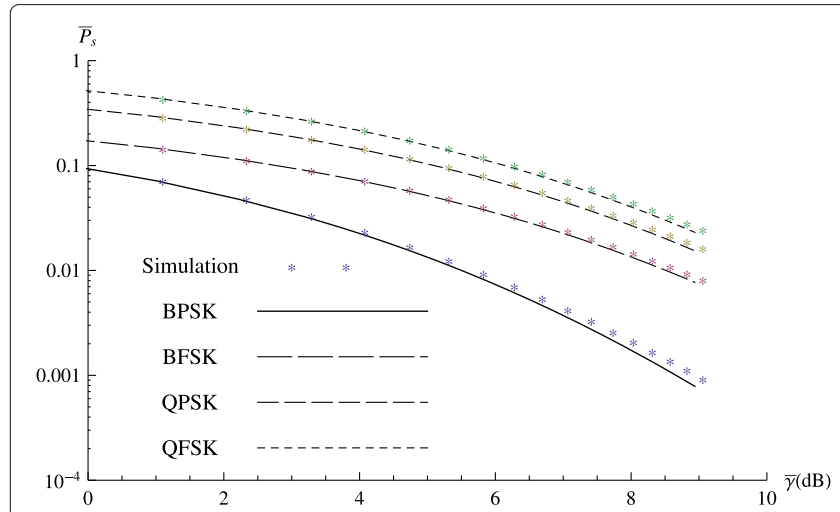

(a) ASEP : $\varphi=0.223, \beta=3$

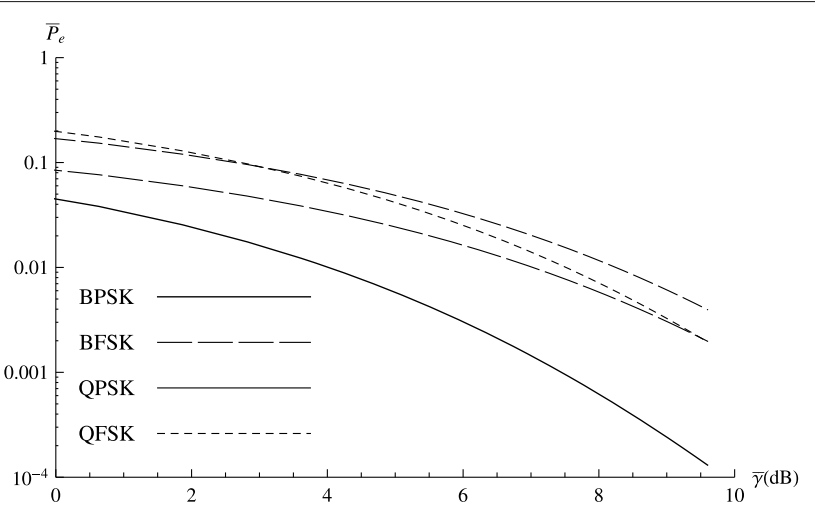

(b) ABEP : $\varphi=0.225, \beta=4$

Fig. 15 Average error probability of various modulations for 3-branch EGC receiver over i.n.i.d WFC

Figure 9 shows both analytical and simulated ORA capacities versus $L$ for EGC receiver, plotted respectively from (34), and via Monte Carlo simulation from (35). It can be seen from the two curves that the approximate analytical expression of ORA capacity is nearly similar to the simulation one. The capacity is plotted for several values of $\beta$, assumed to be the same for each receiver branch, and $\varphi=0.223$. Besides, the greater is $\beta$, the better is the maximal spectral efficiency of the system.

In Fig. 10, the OPRA capacity, given in (37), versus optimal threshold SNR $\gamma_{0}$, computed numerically by solving (44), is traced for several values of $\beta$, and $\varphi=0.227$. From these curves, we can obviously observe that below $\gamma_{0}=0.2$, no data can be transmitted. Moreover, the greater is $\beta$, the higher is optimal simultaneous power and rate adaptation capacity.

Figure 11 plots both the simulated and the analytical OPRA capacity versus $L$ for $\varphi=-0.225$ and several values of $\beta$, expressed in (36) and (37), respectively. We can notice that the greater is $L$, the higher is the capacity under OPRA police. Furthermore, as the shape parameter $\beta$ increases, the capacity improves.

Figure 12 depicts the OPRA capacity, noted in (37), versus $\varphi$ for $L=3$, and many values of $\beta$. We can conclude that the lower is $\varphi$, the higher is the capacity. In addition, when the parameter $\beta$ increases, the OPRA capacity becomes greater.

Figure 13 shows both analytical and simulated CIFR capacity versus $L$ under MRC receiver, plotted respectively from (46), and via Monte Carlo simulation from (47), for $\varphi=0.223$ and several values of $\beta$. What is clear from all curves is that the approximate analytical expression of CIFR capacity is closed to the simulated one. It can be also seen that the greater is $\beta$, the better is the capacity.

In Fig. 14, the capacity under TCIFR policy, given in (49), is traced versus $L$ for $\beta=2.8, \varphi=-0.223$, and several values of a fixed cutoff fade depth $\gamma^{*}$. This capacity is simulated via Monte Carlo statistical method using (50). It is obvious that the smaller is $\gamma^{*}$, the better is TCIFR capacity. Moreover, the diversity order $L$ increases gradually the TCIFR capacity regardless of $\gamma^{*}$.

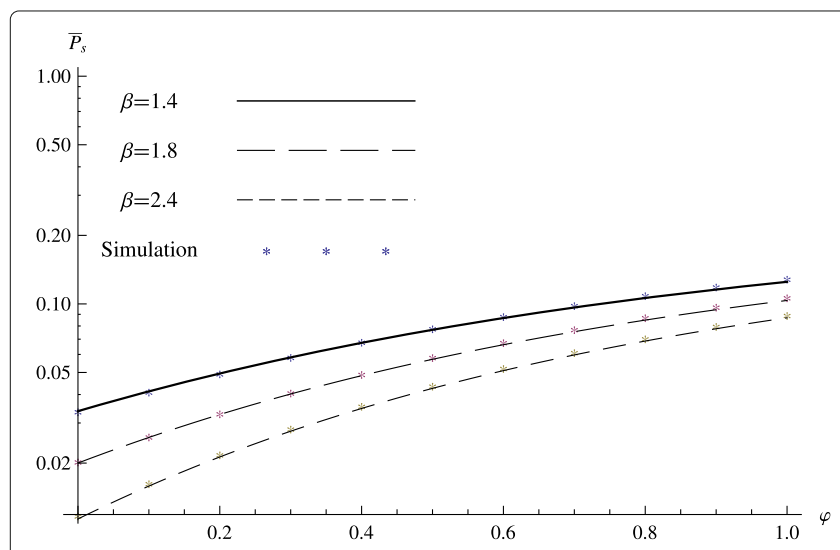

(a) BPSK

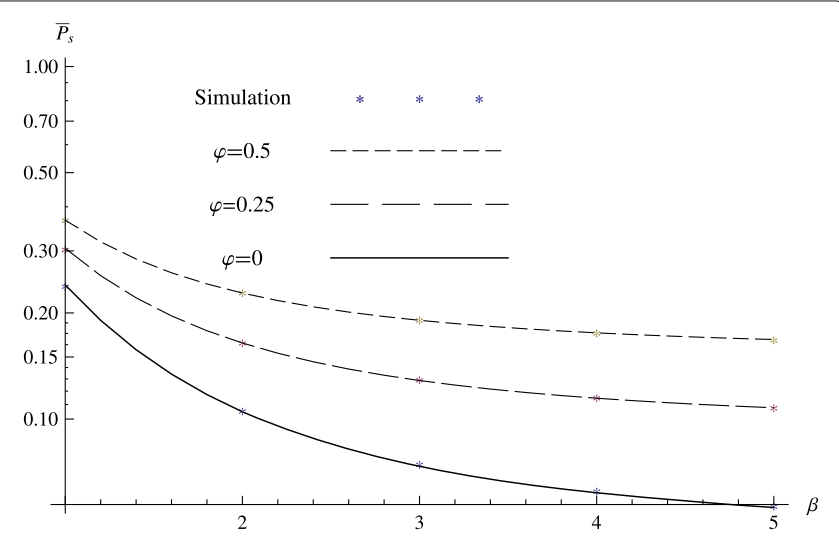

(b) QPSK

Fig. 16 Average symbol error probability of BPSK and QPSK modulations for 4-branch EGC receiver over i.n.i.d WFC 
Figure $15 \mathrm{a}, \mathrm{b}$ depicts the simulated and the approximate ASEP and ABEP, given in (51) and (55), respectively. The curves are plotted versus average output SNR (in dB) using the values of parameters summarized in Table 1 of both $M$-FSK and $M$-PSK ( $M=2$ and $M=4$ ) modulation schemes for 3-branch EGC receiver over i.n.i.d WFC. What is clear from these figures is that QFSK improves the system performance considerably compared to the rest of schemes over entire range of $\bar{\gamma}$. Additionally, average error probability decreases as $\bar{\gamma}$ increases. Besides, the analytical and the simulated curves are confounded.

Figure 16a, b shows the simulated and the approximate ASEP, given in (51), versus $\varphi$ and $\beta$ respectively. The curves are plotted, using the parameter values summarized in Table 1 , for $M$-PSK $(M=2$ and $M=4)$ modulation schemes and 4-branch EGC receiver over i.n.i.d WFC. We can notice that the smaller is $\varphi$, the greater is the output SNR; therefore, the ASEP becomes small. Moreover, the analytical curves match the simulation ones, which proves the accuracy of the proposed approximation.

\section{Conclusions}

In this paper, we have derived a new tight approximation for the PDF of output SNR at EGC receiver operating under uncorrelated but not identical Weibull fading channels. Based on this approximate PDF, the analytical expressions of many performance criteria have been derived. All results are illustrated using Mathematica software and validated by Monte Carlo simulation. The results that we have achieved are far better than all previous works.

\section{Competing interests}

The authors declare that they have no competing interests.

Received: 24 August 2016 Accepted: 8 December 2016

Published online: 03 January 2017

\section{References}

1. MK Simon, M-S Alouini, Digital communication over fading channels, 2nd edn. (Wiley, New York, 2005)

2. VA Aalo, Performance of maximal-ratio diversity systems in a correlated Nakagami-fading environment. IEEE Trans. Commun. 43(8), 2360-2369 (1995)

3. P Lombardo, G Fedele, MM Rao, MRC performance for binary signals in Nakagami fading with general branch correlation. IEEE Trans. Commun. 47(1), 44-52 (1999)

4. J Luo, JR Zeidler, S McLaughlin, Performance analysis of compact antenna arrays with MRC in correlated Nakagami fading channels. IEEE Trans. Veh. Technol. 50(1), 267-277 (2001)

5. NC Beaulieu, A Abu-Dayya, Analysis of equal-gain diversity on Nakagami fading channels. IEEE Trans. Commun. 39(2), 225-234 (1991)

6. A Annamalai, C Tellambura, VK Bhargava, Equal-gain diversity receiver performance in wireless channels. IEEE Trans. Commun. 48(10), 1732-1745 (2000)

7. NC Sagias, GK Karagiannidis, Gaussian class multivariate Weibull distributions: theory and applications in fading channels. IEEE Trans. Inf Theory. 51(10), 3608-3619 (2005)

8. JCS Santos Filho, M Daoud Yacoub, Simple precise approximations to Weibull sums. IEEE Comm. letters. 10(8), 614-616 (2006)
9. GK Karagiannidis, DA Zogas, NC Sagias, SA Kotsopoulos, GS Tombras, Equal-gain and maximal-ratio combining over nonidentical Weibull fading channels. IEEE Trans. on Wireless Comm. 4(3), 841-846 (2005)

10. DA Zogas, NC Sagias, GS Tombras, GK Karagiannidis, Average output SNR of equal-gain diversity receivers over correlative Weibull fading channels. Euro. Trans. Telecomms. 16, 521-525 (2005)

11. F El Bouanani, H Ben-Azza, M Belkasmi, New results for the Shannon channel capacity over generalized multipath fading channels for MRC diversity. EURASIP Jour. on Wirel. Comm. Networking, 336 (2012)

12. F El Bouanani, A new closed-form approximations formulas for MRC receiver over non-identical Weibull fading channel, International Wireless Comm. and Mobile Computing Conference (IWCMC' 14), (Nicosia, 2014), pp. 600-605

13. F El Bouanani, H Ben-Azza, Unified analysis of EGC diversity over Weibull fading channels. Int. Journal Commun. Syst. (2015). https://www.scopus. com/authid/detail.uri?authorld $=55217720500$

14. F El Bouanani, H Ben-Azza, Efficient performance evaluation for EGC, MRC and SC receivers over Weibull multipath fading channel. 10th Intern. Conference on Cognitive Radio Oriented Wireless. Networks (CROWNCOM), pp. 347-357, (2015), Doha

15. A Bessate, F El Bouanani, Performance analysis of MRC diversity in correlated Weibull fading channels, Intern. Conference on Wireless Networks and Mobile Communications (WINCOM'15). (2015), Marrakech

16. GJ Schlenker, Methods for calculating the probability distribution of sums of independent random variables. U.S. army armament, munitions and chemical command systems analysis office rock. Island, (1986), Illinois

17. NC Sagias, GK Karagiannidis, PT Mathiopoulos, TA Tsiftsis, On the performance analysis of equal-gain diversity receivers over generalized gamma fading channels. IEEE Trans. Wirel. Commun. 5(10), 2967-2975 (2006)

18. KP Peppas, HE Nistazakis, GS Tombras, An overview of the physical insight and the various performance metrics of fading channels in wireless communication systems. Wireless Communication Systems. INTECH Open Access Publisher (2011)

19. J Cheng, C Tellambura, NC Beaulieu, Performance analysis of digital modulations on Weibull fading channels. IEEE Trans. on Wireless Comm. 3(4), 1124-1133 (2004)

20. M Abramowitz, IA Stegun, Handbook of mathematical functions with formulas, graphs, and mathematical tables, 9th ed. (Dover Press, New York, 1972)

21. Wolfram (2001). The Wolfram functions site (online) available at http:// functions.wolfram.com. Accessed Mar 2016

22. N Kong, LB Milstein, SNR of generalized diversity selection combining with nonidentical Rayleigh fading statistics. IEEE Trans. Commun. 48, 1266-1271 (2000)

23. MS Alouini, AJ Goldsmith, Capacity of Rayleigh fading channels under different adaptive transmission and diversity-combining techniques. IEEE Trans. Veh Tech. 48(4) (1999)

\section{Submit your manuscript to a SpringerOpen ${ }^{\circ}$ journal and benefit from: \\ - Convenient online submission \\ - Rigorous peer review \\ - Immediate publication on acceptance \\ - Open access: articles freely available online \\ - High visibility within the field \\ - Retaining the copyright to your article}

Submit your next manuscript at $>$ springeropen.com 